\title{
Perceptual and conceptual priming in a semantic reprocessing task
}

\author{
DAN J. WOLTZ \\ University of Utah, Salt Lake City, Utah
}

\begin{abstract}
Two experiments are reported that addressed the relative involvement and nature of perceptual and conceptual priming in a semantically complex task. Both experiments investigated facilitation from repeated semantic comparison trials in which subjects decided whether two words had the same meaning (e.g., moist damp). The first experiment compared the magnitude and persistence of perceptual and conceptual priming components. Perceptual priming effects were modest, and contrary to some previous evidence, they did not appear to be more persistent than nonperceptual priming effects. The second experiment investigated the memory processes involved when perceptual priming was eliminated through a modality change between prime and target trials. Evidence suggested that conceptual priming primarily involved memory for the meaning comparison processes rather than better access to existing memory for the stimulus words.
\end{abstract}

Some researchers have noted that the priming measures used to distinguish implicit from explicit memory performance have been primarily data driven (Blaxton, 1989; Roediger, 1990; Roediger, Weldon, \& Challis, 1989; Srinivas \& Roediger, 1990; Weldon, 1991). The most common measures of implicit memory have been primed word fragment completion, word stem completion, and perceptual word identification. In each of these tasks, a set of words is primed, generally through the presentation of a word list, with processing instructions but with no mention of a subsequent memory test. Then the primed words, along with an equivalent set of unprimed words, are presented in the particular test's format. Priming is indexed by the performance difference between the primed and unprimed words. These tasks appear to have relatively minor semantic or conceptual processing demands. Moreover, enhancement of performance from prior experience appears primarily to be a function of facilitated perceptual reprocessing (Blaxton, 1989; Jacoby, 1983; Weldon, 1993; Weldon \& Roediger, 1987).

The focus of previous researchers on data-driven priming tasks corresponds to the fact that current priming and implicit memory theories emphasize perceptual memory. Most notably, Tulving and Schacter (1990) have proposed that direct priming phenomena are represented

This research was supported in part by a grant from the U.S. Air Force Office of Scientific Research Cognition Program (F4962093-1). The experiments were conducted at the facilities of Armstrong Laboratory in collaboration with the Learning Abilities Measurement Program. I wish to thank Vivien Bainbridge, Keith Horton, Patrick Kyllonen, and Mary Sue Weldon for helpful suggestions regarding previous versions of the paper. 1 also acknowledge Trace Cribbs, Cindi Garcia, Janice Hereford, and Rich Walker for their computer programming efforts. Correspondence concerning this article should be addressed to D. J. Woltz, Department of Educational Psychology, $327 \mathrm{MBH}$, University of Utah, Salt Lake City, UT 84112 (e-mail: woltz@gse.utah.edu) in a distinct memory system, the perceptual representation system (PRS). Although Tulving and Schacter briefly acknowledge that nonperceptual or conceptual priming may occur, they devote little attention to it. For example, they define priming as "a nonconscious form of human memory, which is concerned with perceptual identification of words and objects" (p. 301).

Despite the preponderance of perceptual priming research, conceptual priming has been clearly demonstrated in tasks that conform to definitions of implicit memory measurement. Blaxton (1989) demonstrated that general knowledge test performance (i.e., answering questions such as What is the fastest animal on earth?) showed priming effects from prior exposure to the answers in another context. Moreover, she showed that priming in the general knowledge test was enhanced more by generating the primes from semantic cues, whereas priming in a word fragment completion task was enhanced more by reading the primes (cf. Gardiner, 1988). Hamann (1990) found a similar level of processing effect on priming in the general knowledge test.

Researchers have also demonstrated conceptual priming in the category generation task (e.g., the subject is asked to name exemplars for a category such as furniture, and the likelihood of generating specific exemplars is affected by prior processing). Srinivas and Roediger (1990) showed that generating target words rather than reading them in a study phase produced greater facilitation in this task, but not in word fragment completion. Rappold and Hashtroudi (1991) and Hamann (1990) both manipulated the level of processing during the study phase in other ways, and they found similar results. Finally, Smith and Branscombe (1988) reported a generation effect in a task that required the production of a category label (a behavioral trait) in response to exemplars (behaviors).

In contrast to the evidence for perceptual priming, these demonstrations of conceptual priming have oc- 
curred in tasks with notable semantic processing demands. Unfortunately, the available evidence about priming in semantically complex tasks is quite limited. Beyond demonstrations that conceptual priming exists, little is known about the specific nature and relative contributions of perceptual and conceptual priming within them. Much of the evidence that does exist regarding these issues comes from research done with two semantically complex priming tasks, text reprocessing and repeated semantic comparisons.

\section{Text Reprocessing}

Research on rereading text has produced evidence for both perceptual and conceptual priming components. However, the evidence is somewhat mixed, and the interpretations vary. Evidence showing a contribution of data-driven priming in rereading has come primarily from studies in which the perceptual demands of reading were increased with the use of mirror image text (e.g., Kolers, 1975, 1976; Masson, 1986), and from studies in which the modality of passage presentation was varied across prime and target text occurrences (e.g., Levy \& Kirsner, 1989). In contrast, most single-modality studies on rereading facilitation done with normal text have yielded weak or nonexistent perceptual priming effects (Carr, Brown, \& Charalambous, 1989; Levy \& Kirsner, 1989). The latter findings are consistent with those from other reading-related repetition priming tasks such as lexical decision (Brown, Sharma, \& Kirsner, 1984; Scarborough, Cortese, \& Scarborough, 1977) and tachistoscopic word identification (Morton, 1979).

One study has deviated from the pattern of results just described. Jacoby, Levy, and Steinbach (1992) found clear evidence for perceptual priming in the rereading of normal text. They found faster rereading times when visual details (i.e., font) remained unchanged between reading and rereading. However, they found this effect only when subjects read text silently for the purpose of answering questions. They postulated that data-driven memory processes play a role primarily when reading is backgrounded in relation to some other purpose (i.e., question answering), and that under such circumstances, reading performance relies more on episodic (or instance) memory traces that require perceptual specificity to be invoked.

Evidence for conceptually driven priming in rereading has also been found, but again, the nature of this priming is not well understood. Carr et al. (1989) found that oral rereading facilitation was insensitive to changes in the linguistic structure of sentences from reading to rereading episodes (i.e., scrambled vs. coherent sentences). This, combined with their finding reported earlier that typography did not affect rereading facilitation, led them to argue that rereading facilitation might be due solely to priming at the level of abstract word representation (cf. Levy \& Kirsner, 1989; Whittlesea, 1990).

In contrast to Carr et al. (1989), others have found evidence that rereading facilitation may in part depend on repeated comprehension processes. In a variety of experiments, the magnitude of rereading facilitation depended on the degree to which linguistic structure was altered across repetitions (e.g., shuffling paragraphs within passages, shuffling sentences within paragraphs, shuffling clauses across sentences, paraphrasing sentences, and varying syntax). As a whole, these studies suggest that rereading facilitation depends in part on memory at the message or proposition level of representation (Levy, Barnes, \& Martin, 1993; Levy \& Burns, 1990; Masson, 1993).

\section{Repeated Semantic Comparisons}

Semantic comparison is another verbal task that is generally believed to involve both data-driven and conceptually driven processes (see Jackson \& McClelland, 1979; Kyllonen, Tirre, \& Christal, 1991; Woltz, 1990b). On a semantic comparison trial, two words (e.g., moist damp) are presented; subjects must decide whether the words are synonyms or are unrelated. When exact repetitions of such comparisons are built into the trial blocks at various lags, priming is evidenced by reduced response latency on the repeated trials relative to new or nonrepeated trials. In previous studies done with this task, trials repeated after relatively short delays have produced latency savings of 200-500 msec (Woltz, 1988, 1990a, $1990 \mathrm{~b})$. Moreover, trials repeated after 8 days still exhibit latency savings of approximately $100 \mathrm{msec}$ relative to new trials (Woltz \& Shute, 1993, 1995).

Previous research with this task has revealed a datadriven component in priming without the use of perceptually complex typography or cross-modal priming. Substantial and apparently long-lasting effects of case change between prime and target trials were found with the use of standard upper- and lowercase characters on a computer display (Woltz, 1990b). Jacoby et al. (1992) noted that this finding is consistent with their notion that instance-specific, data-driven memory processes play a role in priming during text processing when reading is backgrounded in relation to some other purpose (i.e., meaning comparison in this case).

Although the evidence supported the existence of a data-driven priming component in the semantic comparison task, it represented only $10 \%-15 \%$ of the total priming effect (Woltz, 1990b). The $85 \%-90 \%$ of priming not attributable to perceptual consistency presumably was due to priming conceptual processes, but the evidence was inconclusive as to the exact nature of this conceptual priming. Specifically, priming of abstract meaning representations in semantic memory did not appear to be instrumental in the long-lasting repetition priming effects. That is, primes that contained word pairs that were semantically related to, but lexically different from, the target trial words produced only short-lived facilitation (e.g., the prime trial big large produced only temporary facilitation for huge gigantic but long-lasting facilitation for a repeat of big large).

These results resemble those from research on the reprocessing of text. Similar to some of the reading evidence (e.g., Jacoby et al., 1992) is the support for a datadriven component in the priming of semantic comparisons. 
There also appears to be a conceptually driven component in the priming of semantic comparisons. However, as in the text reprocessing research, it is not clear what memory processes and representations underlie conceptual priming. Consistent with the proposal by Carr et al. (1989), it could be the activation or strengthening of lexical memory units. Alternatively, consistent with the findings of Levy et al. (1993) and Masson (1993), the priming effects could depend on memory for specific conceptual processing components of the priming event, such as meaning comparison and decision.

The two experiments reported here were performed in order to address questions regarding the joint influence of data-driven and conceptually driven processes in repetition priming of semantic comparisons. Priming effects in this and other tasks are long-lived, but it is not clear whether the persistence is due to data-driven or conceptually driven memory components, or both. Experiment 1 addressed the question of whether there are differences in the longevity of data-driven and conceptually driven priming components. The second experiment addressed questions regarding the specific conceptually driven processes that might be involved in the repetition priming. In this experiment, the involvement of abstract memory for individual words (i.e., lexical memory units that are common across different encoding modalities) was contrasted with the involvement of memory for specific word meaning comparisons.

\section{EXPERIMENT 1}

Priming effects lasting over 1 year have been reported for reading inverted text (Kolers, 1976) and for performance on word fragment completion tasks (Sloman, Hayman, Ohta, Law, \& Tulving, 1988). Tulving and Schacter (1990) suggest that these remarkably persistent memory effects reflect the PRS, implying that it is the data-driven component of priming that is long lasting. Furthermore, a few investigations of conceptually driven priming over varying time delays suggest that this form of priming may be relatively short lived. Shimamura and Squire (1984) found that amnesics did not differ from normals in a primed free association task, but that performance for both groups reached the baseline level (i.e., unprimed performance) within 2 h. Similarly, Hamann (1990) found that primed category exemplar generation performance approached the baseline level within $90 \mathrm{~min}$.

In sum, previous evidence about forgetting within priming tasks suggests that perceptual priming may be more persistent than conceptual priming. However, none of these studies contrasted the forgetting of perceptual and conceptual priming components within the same task. Consequently, the observed forgetting differences may have reflected task attributes beyond the data- versus conceptually driven process distinction.

To test whether conceptual priming is less persistent than perceptual priming, the effect of perceptual consistency in repeated semantic comparison trials was investigated over a range of delays between prime and target trials. Given the previous findings with this task, perceptually consistent repetitions (i.e., identical typography in prime and target trials) should produce both dataand conceptually driven priming. In contrast, perceptually inconsistent repetitions (i.e., different typography in prime and target trials) should produce primarily conceptually driven priming. Given these assumptions, the perceptually consistent repetitions should always yield priming effects that are greater than or equal to the inconsistent repetitions. However, if conceptual priming is short lived relative to perceptual priming, the priming effects for perceptually inconsistent repetitions (i.e., involving little or no perceptual priming component) should show greater decline over lag intervals.

A second question addressed by this experiment pertained to the relative magnitude of the data-driven priming component. In earlier research on the perceptual priming component in this task, case change was used in order to manipulate perceptual consistency (Woltz, $1990 \mathrm{~b}$ ). This may have led to an underestimation of the data-driven component, because many perceptual features of words are maintained across changes in case. So, perceptual consistency was manipulated in the present experiment by changing case, font, and size attributes of prime and target stimulus words. The question of interest was whether the effect of perceptual consistency would remain small in relation to the total priming effect.

\section{Method}

Subjects. The subjects were 508 U.S. Air Force recruits in their 11 th day of basic training at Lackland Air Force Base, TX. Approximately $6 \%$ of these subjects were eliminated because of performance scores that indicated a lack of effort (i.e., high error rates or failure to complete the experimental tasks). Of the remaining 477 subjects, 406 were male and 71 were female. All subjects were high school graduates, and approximately $25 \%$ had some college course work. The age of Air Force recruits ranges from 17 to 27.

Apparatus. The experimental task was administered on Zenith Z-248 microcomputers with standard keyboards and EGA color video monitors. Materials were presented on the monitors in $24 \times$ 80 character text mode. Software was written to achieve millisecond timing of response latency recording (Walker, 1985).

Materials. Three hundred twenty stimulus sets were used in the experimental trial conditions. Each stimulus set contained a stem word, a synonym of the stem for positive match trials, and an unrelated foil for negative match trials (e.g., moist, damp, biue). The stimuli had been used in previous research (Woltz, 1988, 1990a, 1990b; Woltz \& Shute, 1993), and they were selected so that average error rates approximated $10 \%$ or less under instructions that emphasized response speed. There was a separate pool of 72 comparable stimulus sets for warm-up trials. For each subject, stimuli from these two stimulus pools were randomly assigned to the various trial conditions described below.

Procedure. The subjects were tested in groups of 30-40, with each subject at an individual testing carrel containing a microcomputer. Each subject participated for $3.5 \mathrm{~h}$ in a series of experimental tasks. Data from the first experimental task are reported here.

At the beginning of each session, the subjects were given a general orientation to the experimental session and practice in locating keys on the computer keyboard. All instructions were computer administered, and proctors were available to answer questions. Following the initial orientation, each subject performed the semantic comparison task. 
The semantic comparison task consisted of 12 blocks of 54 semantic comparison trials, where some trials were new and some were repetitions of previous trials. On each trial, two familiar words were presented at the center of the display until the subject responded " $\mathrm{L}$ " (for like) or " $\mathrm{D}$ " (for different) according to whether the words' meanings matched. The two words in each trial were presented one above the other, separated by one blank line in the 24-line computer display. Each trial was preceded by an attention cue (one asterisk presented at the center of where the two words would appear) for $1,000 \mathrm{msec}$, followed by a blank screen for $500 \mathrm{msec}$.

The subjects were instructed to respond as quickly as possible without making errors. Response feedback was designed to encourage both speed and accuracy. An individual's trial response latency was presented for $1,000 \mathrm{msec}$ following correct responses. The word WRONG, a low tone, and the message, BE MORE CAREFUL followed errors for 4,000 msec. A blank screen followed all feedback frames for $500 \mathrm{msec}$. After each block of 54 trials, the subjects received a brief rest and were shown their average latency and percent correct for those trials (instructions regarding speed and accuracy were also repeated).

Half of all the trials in each block were positive matches (e.g. moist damp), and half were negative (e.g., moist blue). Half of all the trials in each block were presented in one character style, and the others were presented in a different character style. One character style consisted of all lowercase letters in small triplex font. The letters were stretched horizontally relative to their vertical size and were approximately $6 \mathrm{~mm}$ in height and ranged from 4 to $13 \mathrm{~mm}$ in width. The other character style consisted of all uppercase letters in large small font. These letters were stretched vertically relative to their horizontal size and were approximately $22 \mathrm{~mm}$ in height and ranged from 4 to $6 \mathrm{~mm}$ in width.

Repetitions were placed within and across trial blocks in such a way that each subject had 64 repeated trials at each of four lags. Repetition lag was crossed with match type (positive vs. negative), character style, and consistency of character style across first and second occurrence (i.e., half of all repeated trials at each lag had the same character style as its first occurrence, and half had the alternative character style). Repeated trials occurred at lags of $1,8,54$, and 432 trials.

The manipulation of repetition lag was accomplished as follows. Each of the 12 trial blocks began with 6 warm-up trials. Of the remaining 48 trials in each block, there were four Lag 1 repetitions (no trials intervening between first and second occurrence) and four Lag 8 repetitions ( 7 trials intervening between first and second occurrence), except in Blocks 5-8, which contained eight Lag 1 and Lag 8 repetitions. Lag 54 repetitions were contained exclusively in Blocks $2,4,6$, and 8 . That is, each even-numbered block contained 16 repetitions of first-occurrence trials that had been presented in the previous odd-numbered block. Lag 432 repetitions were contained exclusively in Blocks 9-12. Each of these blocks contained 16 repetitions of first-occurrence trials that had been presented in Blocks 1-4. In addition, Blocks 9-12 each contained 16 new (nonrepeated) trials. Trials within each block were randomly ordered for each subject within the constraints imposed by the fixed repetition lags.

\section{Results and Discussion}

Both error and latency data from this study were analyzed, because both have shown priming effects in previous research (e.g., Woltz, 1990b). However, response latency was seen as the primary index of priming. The multivariate approach to repeated measures analysis of variance (ANOVA) was used to test the hypotheses within each dependent measure (O'Brien \& Kaiser, 1985). For multivariate tests, approximate $F$ ratios are reported in lieu of multivariate statistics because of their greater familiarity. The $p$ value for all statistical tests was set at .05 .

Latency. Figure 1 shows the latency mean data for first- (new) and second- (repeated) occurrence trials by match type and lag (error bars represent $95 \%$ confidence intervals appropriate for repeated measures). The firstoccurrence means associated with each lag were com-
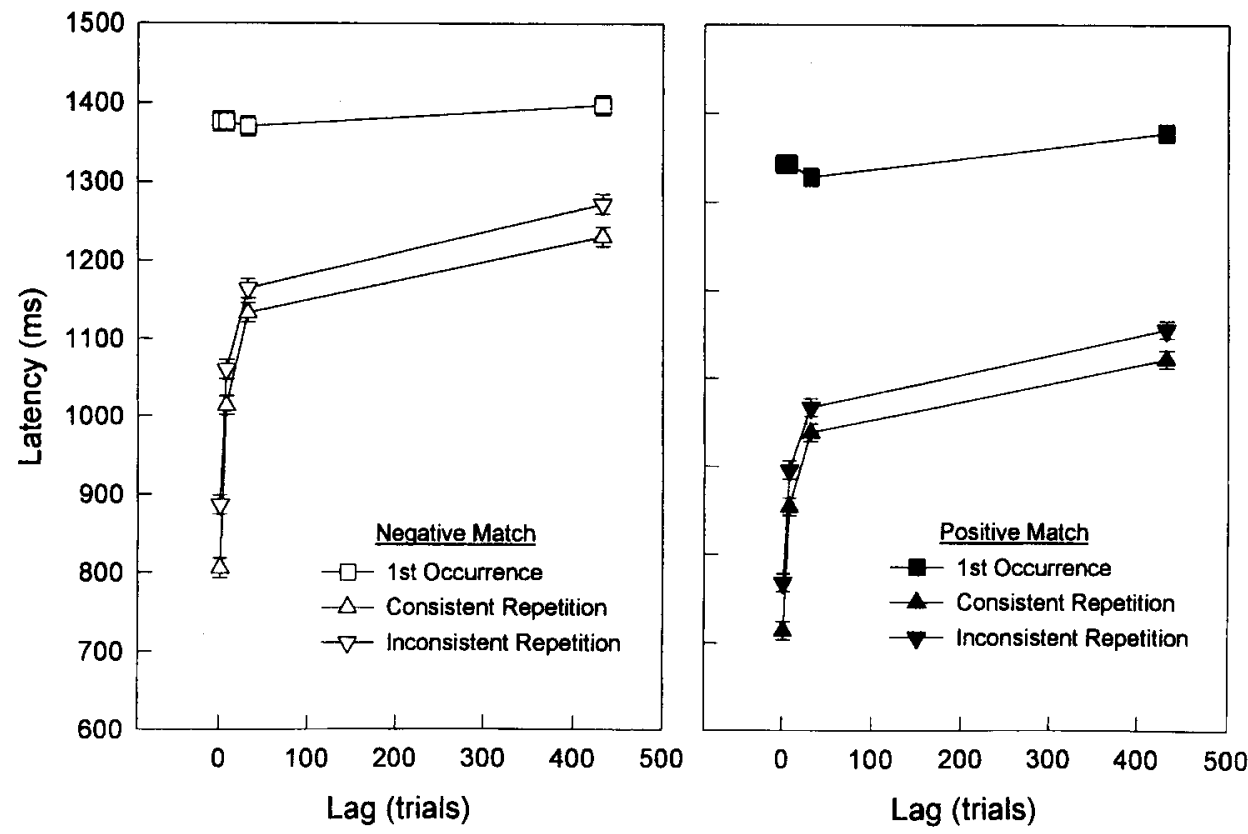

Figure 1. Mean latency data from Experiment 1 by match type, occurrence, and lag (error bars represent $95 \%$ confidence intervals for repeated measures). 
puted on all new trials within the trial blocks where the repeated trials occurred. For example, second-occurrence trials for Lag 432 were contained in Blocks 9-12, so firstoccurrence trials in Blocks 9-12 were used as Lag 432 first-occurrence trials for computing priming effects (not the actual first-occurrence trials from Blocks 1-4).

Note in Figure 1 that first-occurrence latency differed by match type. Positive match response latency was less than negative match latency $\left[F(1,476)=864.30, M S_{\mathrm{e}}=\right.$ $14,839.60]$. This difference is consistent with previous evidence on the performance of semantic comparisons (Woltz, 1990a, 1990b). There was also a significant block (lag) $\times$ match type interaction in the first-occurrence latency data $[F(2,475)=11.62]$. Response latency to both positive and negative match trials increased over trial blocks probably because of fatigue, but this increase was slightly greater for positive match trials.

Ideally, first-occurrence latency would have been equivalent across all three trial block sets. However, the performance differences were relatively small. Furthermore, priming effects were computed within trial block set in an attempt to control for these differences.

Priming was computed for the various trial types as the difference between second-occurrence (repeated) median latency and first-occurrence median latency within match type, lag, and trial block. The priming effects can be seen in Figure 1 by comparing the mean data for firstand repeated-occurrence trials.

As can be seen in Figure 1, priming effects were slightly greater for repeated positive trials than for repeated negative trials $\left[F(1,476)=84.68, M S_{\mathrm{e}}=2,905,332\right]$. Across all other trial conditions, there was a mean priming effect of $M=342 \mathrm{msec}(S D=130)$ for positive trials and $M=303 \mathrm{msec}(S D=154)$ for negative trials. Also evident in Figure 1 is that priming effects in both match types decreased with longer lags $[F(3,474)=$ $812.61]$. Finally, priming effects were greater for consistent repetitions that preserved the original text characteristics of primes $\left[F(1,476)=300.77, M S_{\mathrm{e}}=12,900\right]$. All three of these findings were consistent with the priming effects found in previous research (Woltz, 1990b).

The finding that priming was greater for perceptually consistent repetitions directly reflects the data-driven priming component in this task. As the ANOVA indicated, the difference between perceptually consistent and inconsistent repetitions ( $M=45.10 \mathrm{msec}, S D=56.79$ ) was quite dependable. However, as in previous work (Woltz, 1990b), this perceptual consistency effect constituted less than $15 \%$ of the total identity priming effect, which averaged over $300 \mathrm{msec}$ across conditions. Thus, the data-driven component of priming in this task appeared to be relatively minor despite greater orthographic discrepancy between first- and second-occurrence trials.

The second question of interest was whether there was more forgetting in perceptually inconsistent trials that presumably had little or no perceptual priming component. Although the difference between the consistent and inconsistent repetitions appears fairly constant in Figure 1 , there was a significant perceptual consistency $X$ lag interaction $[F(3,474)=18.67]$. However, the direction of this interaction is opposite what would be expected if conceptual priming lacked persistence. The effect of perceptual consistency decreased rather than increased across the lags investigated.

As noted by several researchers, conclusions drawn from the comparison of forgetting rates when there are different initial levels of learning (in this case, priming) requires an assumption that the dependent measure is linearly related to the relevant psychological dimensionin this case, changes in memory strength (e.g., Bogartz, 1990; Loftus, 1985; Slamecka, 1985; Wixted, 1990; Wixted \& Ebbesen, 1991). Such an assumption is difficult to justify, and there appears to be no easy way to resolve this scaling dilemma. It is possible, however, to conclude that the data are incompatible with the prediction that the perceptual consistency effect (the difference between consistent and inconsistent repetition priming) will increase over lags because only the perceptually consistent trials will show long-lasting priming. This conclusion rests only on the assumption that the current dependent measure representing priming is monotonically related to the psychological construct of interest (i.e., memory strength).

Errors. Figure 2 presents the mean error data for firstand second-occurrence trials by match type and lag (the error bars represent $95 \%$ confidence intervals appropriate for repeated measures). As can be seen in this figure, the errors on positive first-occurrence trials exceeded the errors on negative first-occurrence trials $[F(1,476)=$ $\left.492.69, M S_{\mathrm{e}}=31.67\right]$. One explanation for this is that some positive match word pairs may have appeared to be unrelated because a stimulus word had multiple senses (e.g., block obstruct). Also evident in Figure 2 is that errors varied somewhat across the block sets, as is reflected in a significant block set $\times$ match type interaction $[F(2,476)=38.55]$. Errors on positive match trials increased slightly across block sets, while errors on negative match trials decreased slightly. This suggests that subjects' response biases shifted modestly toward negative responding over trial blocks.

As with the latency data, priming was computed for the various trial types as the difference in error rate between repeated- and first-occurrence trials of the same match type within lag and trial block set. As can be seen in Figure 2, error savings were greater for repeated positive trials than for repeated negative trials $[F(1,476)=$ $\left.962.45, M S_{\mathrm{e}}=85.72\right]$. This is consistent with previous evidence (Woltz, 1990a, 1990b). Forgetting was also evident, as error savings decreased with longer repetition lags $[F(3,474)=101.76]$. Finally, there was a small but statistically significant effect of perceptual consistency on error savings $\left[F(1,476)=15.56, M S_{\mathrm{e}}=24.87\right]$. As can be seen in Figure 2, there were greater error savings for perceptually consistent than for perceptually inconsistent repetitions. However, none of the interactions was statistically significant, including the perceptual consistency $\times$ repetition lag interaction. Thus, when forgetting is defined by changes in error rate over the repeti- 

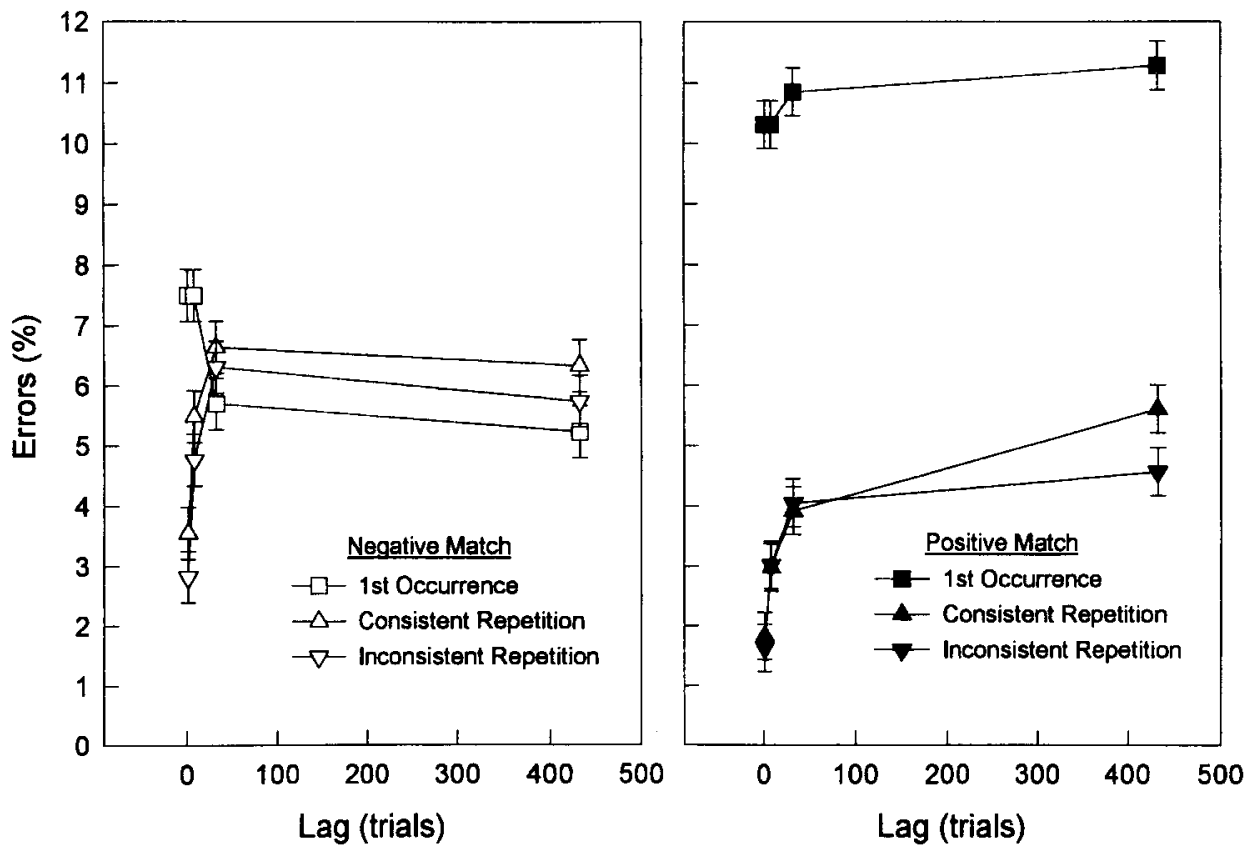

Figure 2. Mean error data from Experiment 1 by match type, occurrence, and lag (error bars represent $95 \%$ confidence intervals for repeated measures).

tion lags investigated here, there is no evidence for greater persistence in the data-driven priming component than in other components.

In total, these results suggest that the role of withinmodality data-driven processes is relatively minor in this priming task. Furthermore, the latency data suggested that the involvement of data-driven processes in priming declines over the lag intervals investigated. Such evidence is inconsistent with the position that the perceptual priming component is primarily responsible for previously observed long-term priming effects in this task.

\section{EXPERIMENT 2}

In Experiment 1, the existence of priming in conceptually driven processes was inferred from the inability of manipulations affecting data-driven processes to account for all of the priming effects. As with much of the evidence for conceptual priming, the mechanisms underlying the nonperceptual priming were not clear. The purpose of this experiment was to test the involvement of particular conceptually driven processes in the repetition priming effects for semantic comparisons.

The approach taken in Experiment 2 was to more fully eliminate data-driven or perceptual components of repetition priming, and then manipulate task characteristics that correspond to specific conceptually driven processes. Changes in priming corresponding to these manipulations were assumed to reflect the contribution of these conceptually driven processes to priming.

In Experiment 1, perceptual consistency was manipulated within a single sensory modality by altering orthographic features of the stimuli. However, with the use of this manipulation, even the inconsistent repeated trials may have shared some perceptual features with their primes, and therefore a proportion of the savings on these trials may have reflected perceptual memory. Furthermore, as described earlier, research on text reprocessing and other priming tasks has shown that crossmodal manipulations produce greater reductions in priming than do feature manipulations within the visual modality. This suggests that modality change may be necessary to fully remove data-driven priming components. In the present experiment, cross-modal priming was investigated with an auditory presentation of the initial stimuli, and repeated trial facilitation was measured in subsequent performance on visually presented trials.

On the presumption that some degree of cross-modal priming would occur, the question addressed in this experiment was whether it would primarily reflect memory changes to abstract lexical units corresponding to the words in each trial, or memory for processes employed in comparing the word meanings. As described earlier, Carr et al. (1989) concluded that priming effects in rereading text were due to activation of lexical memory units. However, this conclusion has been countered by evidence of priming effects associated with passage comprehension processes rather than individual word reading (Levy et al., 1993; Levy \& Kirsner, 1989; Masson, 1993).

The method for contrasting the lexical versus meaning comparison contributions to conceptually driven priming was as follows. As in Experiment 1, there were firstoccurrence and second-occurrence (repeated) trials. However, on some of the first-occurrence prime trials $(33 \%)$, two identical words (e.g., moist moist) were pre- 
sented. These trials will be referred to as lexical primes, because the correct response for positive match trials required subjects only to encode a single word twice and recognize that the two words were identical (i.e., lexical comparisons were required). On the remaining firstoccurrence prime trials $(66 \%)$, two different words were presented for meaning comparison, as in Experiment 1 (e.g., moist damp). These will be referred to as semantic primes (i.e., semantic comparisons were required).

In both semantic and lexical primes, there was a stimulus onset asynchrony (SOA) of $750 \mathrm{msec}$ between the first and second stimulus words. The SOA was included so that subjects could not detect initially whether a trial required a semantic or a lexical comparison. It was assumed that under these conditions subjects would process the first stimulus word in both lexical and semantic prime trials by retrieving its meaning and preparing to compare it to another word's meaning. ${ }^{1}$ Given this assumption, performance on lexical and semantic primes would have involved the same word encoding, meaning retrieval, and response processes. They would have differed primarily in the type of comparison made. Only in the semantic primes would subjects have engaged in the identical comparison of word meanings that would be required of them in later target trials.

Corresponding to the semantic and lexical primes, there were two types of target trials. Some target trials repeated semantic primes exactly. However, because priming trials were auditory and target trials were visual, priming effects in these repetitions were presumed to reflect only conceptually driven processes. Other target trials represented the combination of two lexical primes. For example, the lexical primes large large and big big would have been combined to form the target trial large big. If latency savings on such lexically primed target trials was as large as savings on semantically primed trials, this would suggest that the conceptually driven priming component for this task was at the level of lexical and possibly general semantic memory representation. That is, priming would reflect increased accessibility to the words and perhaps their meanings. However, if the semantically primed target trials had greater savings than the lexically primed trials had, this would suggest that some or all of the conceptually driven priming involved memory for trial-specific semantic processes involved in comparing the word meanings. ${ }^{2}$

\section{Method}

Subjects. The subjects were 238 U.S. Air Force recruits in their 11 th day of basic training at Lackland Air Force Base, TX. Approximately $12 \%$ of these subjects were eliminated because of performance scores that indicated a lack of effort (i.e., high error rates or failure to complete the experimental tasks). Of the remaining 210 subjects, 166 were male and 44 were female. All subjects were high school graduates and approximately $29 \%$ had some college course work.

Apparatus. The semantic comparison priming trials were administered by a Digital Equipment Corporation DEC-Talk speech synthesis device attached to a Zenith Z-248 microcomputer. The semantic comparison target trials were administered on Zenith $Z$ 248 microcomputers as described for Experiment 1.

Materials. The stimuli consisted of 588 stimulus sets similar to those used in Experiment 1 (i.e., a stem word, a synonym of the stem used for positive trials, and a word unrelated to the stem used for negative trials). For each experimental session (described below), a random assignment of stimuli to trial condition was made.

Procedure. The subjects were tested in groups of $30-40$. Each subject participated for $3.5 \mathrm{~h}$ in a series of experimental tasks. Data from only the first experimental task performed by each subject are reported here.

The semantic comparison task was presented in two parts (A and B). Part A contained 504 priming trials presented in seven blocks of 72 trials each. These priming trials were presented auditorily by the DEC-Talk speech synthesizer. All subjects in an experimental session $(N=30-40)$ sat in a classroom and responded to the same auditory stimuli presented by the DEC-Talk over a speaker placed in the front center of the room.

As in the visual semantic comparison trials of Experiment 1 , each trial in Part A consisted of two words, and subjects had to decide whether they were synonyms or unrelated in meaning. As will be described later, these words could be two distinct words, or they could be the same word presented twice. A trial began with an auditory alerting cue (a tone) for $1,000 \mathrm{msec}$, followed by a silent period of $500 \mathrm{msec}$. Then the trial number was presented, followed by a $1,000-\mathrm{msec}$ silent period. The first word was then presented by the DEC-Talk. Because of difficulty that pilot subjects had in comprehending some isolated words spoken by the speech synthesizer, each stimulus word was pronounced and then spelled by the DEC-Talk. Spelling was presented at the rate of three letters per second. Following the pronunciation and spelling of the first stimulus word in a trial, there was a 750 -msec SOA (i.e., a silent period) before the second word of the stimulus set was pronounced and spelled. This was followed by a $4,000-\mathrm{msec}$ period during which the subjects were to respond. The responses were made on a computer-scorable answer sheet by filling in bubbles with a pencil. The answer sheets had numbered items corresponding to the trial numbers. The subjects used the first two of five columns of response alternatives on the answer sheets. Column 1 was labeled Yes, and Column 2 was labeled $N o$.

Twenty four of the 72 trials in each block were negative meaning matches (i.e., negative semantic primes). Another 24 of the trials were positive meaning matches (i.e., positive semantic primes). The remaining 24 trials in each block did not require meaning matches (i.e., lexical primes). Twelve of these trials were positive lexical primes, (e.g., damp damp), and these represented six sets of semantically related trials (e.g., damp damp and moist moist would constitute one set of semantically related lexical primes). The semantically related lexical primes were combined later in Part B to form semantic comparison target trials (e.g., moist damp). The remaining 12 lexical comparison trials involved a negative match (i.e., negative lexical primes). In order to create a negative lexical comparison that did not involve comparing word meanings, a tone was presented for $1,000 \mathrm{msec}$ as the second stimulus in these trials. The subjects were instructed that the tone could obviously never match the meaning of the first stimulus word, so they were to always respond No on these trials.

The order of semantic and lexical primes was randomized within blocks for each experimental session, with the constraint that related lexical primes could not be adjacent but had to occur within eight trials of one another. Because Part A was administered in a group setting, all the subjects within a session received the same stimuli and no feedback could be provided to individual subjects.

Following all 504 trials of Part A, the subjects took a 10 -min break and moved to a different room. Here the subjects sat in separate testing carrels containing microcomputers. Each subject was 
provided with an initial orientation to the computer and its keyboard and then began performing Part B of the semantic comparison task

Part B contained visually presented trials that were identical to those in Experiment 1 with respect to stimulus format, response requirements, and feedback. There were three blocks of 84 trials each. Half of the trials in each block were positive, and half were negative. Half of both positive and negative trials in each block were repeats of trials from Part $A$, and half were new. Repeats of semantic primes presented the stimulus words in the same order as in Part A (i.e., the first word on top and the second word on the bottom). Repeats of lexical primes consisted of one word from each of two lexical primes in Part A. The lexical prime that occurred first in Part A was the top word and the prime that occurred second in Part A was the bottom word.

Within each block of 84 trials in Part B, 14 trials were exact repetitions of positive semantic primes, 14 were positive combined repetitions of related lexical primes (e.g., large large and huge huge from Part A would be combined to form large huge in Part B), 14 were new positive semantic comparison trials with neither word heard in Part A as a part of either semantic or lexical primes, 14 were exact repetitions of Part A negative semantic primes, 14 were negative combined repetitions of unrelated negative lexical primes (e.g., large $\langle$ tone $\rangle$ and attempt $\langle$ tone $\rangle$ from Part A were combined to form large attempt in Part B), and 14 were new negative semantic comparison trials with neither word heard in Part A as part of semantic or lexical primes. Although each subject within a session received the same stimuli per trial condition, the order of target trials in Part B was randomly ordered for each subject. For each session, there was a new random assignment of stimulus sets to trial conditions.

\section{Results and Discussion}

The use of a speech synthesizer for auditory stimulus presentation had the disadvantage that some words were difficult to understand, despite efforts to spell the words phonetically by using special pronunciation characters in the stimulus file submitted to the DEC-Talk. This difficulty evidenced itself in relatively high error rates for some priming trials in Part A. Although the subjects made relatively few errors on lexical primes $(M=2.38 \%$, $S D=3.41$ for positive lexical primes; $M=0.70 \%, S D=$ 1.51 for negative lexical primes), they made more errors on semantic primes $(M=13.71 \%, S D=6.82$ for positive semantic primes; $M=5.08 \%, S D=4.82$ for negative semantic primes). The particularly high error rate for pos-

Table 1

\section{Response Errors (\%) Means and Standard Deviations by} Trial Condition for Auditorily Primed Semantic Comparisons of Experiment 2

\begin{tabular}{lccccc}
\hline & \multicolumn{2}{c}{ Trial Performance } & & \multicolumn{2}{c}{ Savings } \\
\cline { 2 - 3 } \cline { 5 - 6 } Priming Condition & $M$ & $S D$ & & $M$ & $S D$ \\
\hline None (new trial) & \multicolumn{3}{c}{ Positive Trials } & & \\
Lexical comparisons & 9.27 & 5.35 & & \\
Semantic comparison & 10.98 & 6.08 & & -1.71 & 5.89 \\
& 7.68 & 5.03 & & 1.59 & 6.04 \\
None (new trial) & Negative Trials & & \\
Lexical comparisons & 6.47 & 5.06 & & \\
Semantic comparison & 7.06 & 5.29 & & -.59 & 5.33 \\
\hline
\end{tabular}

Note-Savings was defined as the difference between primed and unprimed (new trial) percent error.
Table 2

Response Latency (in Milliseconds) Means and Standard Deviations by Trial Condition for Auditorily Primed Semantic Comparisons of Experiment 2

\begin{tabular}{lccrrr}
\hline & \multicolumn{2}{c}{ Trial Performance } & & \multicolumn{2}{c}{ Savings } \\
\cline { 2 - 3 } \cline { 5 - 6 } Priming Condition & \multicolumn{1}{c}{$M$} & $S D$ & & $M$ & $S D$ \\
\hline None (new trial) & \multicolumn{2}{c}{ Positive Trials } & & \\
Lexical comparisons & 1,155 & 242 & & \\
Semantic comparison & 1,159 & 259 & -4 & 80 \\
& 1,103 & 255 & 52 & 101 \\
None (new trial) & Negative Trials & & \\
Lexical comparisons & 1,274 & 321 & & \\
Semantic comparison & 1,255 & 297 & 19 & 92 \\
\hline
\end{tabular}

Note-Savings was defined as the difference between primed and unprimed (new trial) latency.

itive semantic primes probably reflected the tendency for subjects to respond negatively when they did not hear one or more of the stimulus words correctly. The failure to understand some of the stimulus words would most likely have reduced the magnitude of priming effects in this experiment. The particularly high accuracy for negative lexical primes reflects the fact that whenever a tone was heard instead of a second stimulus word, subjects simply were to respond negatively regardless of the meaning or clarity of the first word.

The subjects' error rates were more uniform across trial conditions in Part B, but there were still some meaningful differences. Table 1 presents the error rates by trial condition. ${ }^{3}$ As can be seen in Table 1, prime type interacted with match type in affecting error savings $\left[F(1,209)=32.70, M S_{\mathrm{e}}=18.08\right]$. This interaction reflected the fact that the semantically primed positive trial condition was the only condition in which positive error savings occurred $\left[F(1,209)=14.72, M S_{\mathrm{e}}=18.23\right]$. The priming effect was not significantly different from zero for either of the negative trial conditions $(p>.05)$. Positive trials primed by lexical comparisons also differed significantly in error rate from unprimed trials, but in the opposite direction $\left[F(1,209)=17.51, M S_{\mathrm{e}}=17.34\right]$. That is, subjects made more errors on lexically primed positive trials than on new unprimed positive trials.

The finding that greater priming occurred for positive than for negative semantically primed trials was consistent with results from Experiment 1 and previous research (Woltz, 1990a, 1990b). Of primary importance, the finding that the semantic but not lexical primes for positive trials produced error savings suggests that conceptually driven priming in this task may reflect memory for the semantic comparison process more than changes to lexical memory units.

The latency data presented in Table 2 were consistent with the accuracy data. The overall effect of prime type (semantic vs. lexical) on latency savings was significant $\left[F(1,209)=67.18, M S_{\mathrm{e}}=3,120\right]$, reflecting the fact that semantic primes produced greater latency savings than did lexical primes. However, as with the error data, 
the interaction between match type (positive vs. negative) and prime type was also significant $[F(1,209)=$ $\left.32.76, M S_{\mathrm{e}}=3,890\right]$. This reflects the fact that the priming difference between semantically and lexically primed trials was primarily evident in positive match trials.

These findings suggest that memory for the semantic comparison process was integral to the conceptually driven priming component of this task. In trials primed by lexical comparisons, the only overlap in cognitive operations between prime and target comprised word identification and meaning retrieval. In trials primed by semantic comparisons, the overlap also included meaning comparison. The fact that semantic primes produced the greatest savings, albeit only in positive match trials, suggests that memory for trial-specific meaning comparison processes in positive match trials may be a crucial component of conceptually driven priming.

The fact that the semantic-lexical prime difference did not exist in negative match trials can be interpreted in several ways. This could be attributed to the inherent differences in the meaning comparison process for negative and positive match trials. The comparison process for two words with shared semantic features may simply be more memorable than that for two unrelated words. Shulman (1974) reported data on word recall and recognition performance following semantic verification trials that is consistent with this explanation. Words from positive match trials (Is a TWINGE sudden?) were remembered better than words from negative match trials (Is a DUNGEON a scholar?).

However, this explanation does not account fully for the differences seen in the latency data. While it can explain the fact that the semantic-lexical prime difference was greater in positive match trials, it does not explain the fact that within lexically primed trials, negative match trials showed more priming than did positive match trials $\left[F(1,209)=6.71, M S_{\mathrm{e}}=8,227\right]$. Lexically primed negative trials showed significant priming $[F(1,209)=$ $8.91, M S_{\mathrm{e}}=4,263$ ], whereas lexically primed positive trials did not $(p>.05)$.

One likely explanation for this result is that the negative priming trials containing lexical comparisons were unique from all other trials and as such were more memorable. They were unique in that the second stimulus was a low tone that probably came as a surprise, since it occurred in a minority of priming trials $(17 \%)$. The possibility that this stimulus feature made these trials more memorable is supported by findings from another set of experiments in which the effect of lexical versus semantic priming was investigated within the visual modality (Woltz, 1995). In one experiment, lexically primed negative trials were formed by combining words from two positive match prime trials (e.g., large huge and attempt try were combined to form large attempt). In another experiment, lexically primed negative trials were formed by combining words from two negative match prime trials that contained one word and a string of X's (e.g., large $X X X X X$ and attempt $X X X X X$ were combined to form large attempt). The latter priming condition, which rep- resents the visual analogue to the tone used in the present study, produced latency savings that were about $50 \%$ greater than in the former condition. ${ }^{4}$ Thus, unique characteristics in negative priming trials (i.e., tones or X's experienced in only a fraction of the trials) may serve to make them more memorable. It is doubtful, however, that this effect involves explicit memory retrieval (i.e., recalling explicitly that a pair of words comes from priming trials with certain characteristics), because the effect was seen in latency but not error data. If explicit recall of priming trial features were used as a cue for target responses, one would expect to see the opposite pattern.

One other result that deserves discussion is the low overall magnitude of latency savings compared with that found in Experiment 1. In Experiment 1, priming effects for perceptually inconsistent trials were over $200 \mathrm{msec}$ for positive trials and over $100 \mathrm{msec}$ for negative trials at the longest lag (432 trials). The priming effects in the present experiment represent only $20 \%-25 \%$ of those effects. A lower magnitude of savings due to cross-modal rather than unimodal priming is certainly expected from previous research with other priming tasks (Clarke \& Morton, 1983; Jacoby \& Dallas, 1981; Kirsner, Milech, \& Standen, 1983; Kirsner \& Smith, 1974; Roediger \& Blaxton, 1987). However, such a drastic reduction as that found here would argue that the manipulation of text characteristics in Experiment 1 left the majority of datadriven priming effects intact.

There are three reasons why the differences in priming magnitude between Experiments 1 and 2 reflect more than the involvement of modality-specific data-driven processes. First, the average repetition lag in Experiment 2 was considerably longer than even the longest lag used in Experiment 1. The longest lag in Experiment 1 was 432 trials, whereas repeated trial lags in Experiment 2 ranged from 505 to 757 . In addition, the time delay between primes and targets was considerably greater than in Experiment 1. Each priming trial took longer because of the spelling of individual words and the longer interval needed for paper-and-pencil responding, and there was a 10-min break and some computer familiarization tasks (approximately $10 \mathrm{~min}$ worth) inserted between priming and target trials in Experiment 2. Overall, the average delay between primes and targets in Experiment 2 was approximately $90 \mathrm{~min}$, whereas delays ranged from a few seconds to approximately $30 \mathrm{~min}$ in Experiment 1 . Second, the priming method of Experiment 2 altered more than the perceptual encoding processes. Response methods also differed between prime and target trials. Subjects responded by filling in empty circles with pencil and paper in the priming trials, whereas they responded by pressing " $L$ " or " $D$ " on a computer keyboard in the target trials. It is not clear how much of an effect the response difference had, but it probably reduced the overall priming effects compared with those found in Experiment 1 to some extent. Finally, as indicated by relatively high error rates during the priming trials of Experiment 2, some stimuli were difficult to understand as they were presented by the speech synthe- 
sizer. This also would serve to reduce the magnitude of priming relative to that in Experiment 1 . Thus, the relatively small priming effects seen in Experiment 2 probably reflect more than the complete removal of overlap in data-driven processing components between prime and target trials.

In summary, both error and latency data from this experiment were consistent in suggesting two conclusions. First, both measures indicated that there was still priming after both encoding and response processes were radically altered between priming and target trials. This repetition priming was presumed to exclusively reflect conceptually driven memory processes. Second, both error and latency data suggested that the most important component of conceptually driven priming in positive trials of this task was memory for the meaning comparison process, and that changes in abstract lexical memory units was probably not a major contributor.

\section{GENERAL DISCUSSION}

These findings offer evidence for the joint role of dataand conceptually driven processes in repetition priming in a relatively complex semantic processing task. Datadriven priming was evident both from the effect of manipulating orthographic details within visually presented trials and from the effect of changing encoding modality between priming and target trials (auditory to visual). Conceptually driven priming was inferred by the failure of encoding consistency manipulations to eliminate priming completely, and by the effect on priming of manipulating the semantic processing consistency between prime and target trials (i.e., lexical vs. semantic comparisons).

Experiment 1 suggested in two ways that data-driven processes or perceptual memory may play a limited role in repetition priming of semantic comparisons. First, the effect of changing perceptual details of visual stimuli between priming and target trials produced a relatively small reduction in latency savings $(15 \%)$. Second, the perceptual consistency effect declined over the lag intervals investigated. This was contrary to predictions, based on previous evidence, that the conceptual priming component would be temporary relative to the perceptual priming component. If only perceptual priming was persistent, one would expect the difference between perceptually consistent and inconsistent repetitions to increase rather than decrease over time.

Experiment 2 demonstrated that when all overlap in perceptual encoding and response execution was removed between prime and target trials, priming was still evident. Such priming effects were presumed to represent only conceptually driven memory processes. More importantly, in contrasting the effects of primes that demanded lexical versus semantic comparisons, this experiment suggested that conceptually driven priming was primarily due to memory for word meaning comparisons as opposed to word identification or meaning retrieval processes.
The interpretation of these findings as evidence about the nature of conceptual priming is based partly on the assumption that the observed facilitation was independent of explicit recognition of the priming events, as has been the case in most demonstrations of perceptual priming. Little evidence exists regarding this matter for any conceptually driven priming tasks. Our initial research in this area suggests that long-term priming effects in the semantic comparison task (up to 28 days) are stochastically independent of the recognition of priming events (Woltz \& Madsen, 1995). ${ }^{5}$ However, more research is needed in order to completely address this issue in this and other conceptually driven priming measures.

If we assume independence from recognition, one view of the nonperceptual priming demonstrated in these experiments could be that it reflects task-specific memory phenomena that may not be of general interest (i.e., the type of conceptual priming observed here may only occur in tasks that require word meaning comparisons). If so, the question arises as to how representative the semantic comparison task of processing performed in everyday life is. I believe that a compelling argument can be made that this task is at least as representative of commonly used cognitive processes as other more widely used priming tasks. Moreover, given the perceptual nature of most previously studied implicit memory tasks, the examination of priming in more semantically complex tasks such as this seems important.

A more important point, however, regarding priming task comparisons may be that the event-specific nature of conceptual priming observed in the present experiments reflects an important characteristic of implicit memory phenomena that may be common to both perceptual and conceptual priming. Evidence for data-driven priming suggests that the underlying memory representations may be trial specific rather than general. That is, altering minor features of familiar stimuli such as orthographic features in text can measurably reduce the magnitude of priming under certain processing conditions (e.g., Jacoby et al., 1992; Masson \& Freedman, 1990; Woltz, 1990b). Similarly, the conceptual priming in the present experiments appeared to depend on trial-specific memory representations rather than on existing memory that is more general in character, such as lexical units or semantic feature representations.

Finally, research findings and theory regarding implicit memory have been viewed by some as the most important breakthrough in memory research of this century (Schacter, 1992). However, the importance of this area of research may ultimately depend on the range of human behaviors and performance abilities that the resulting theories can explain. If the definition of implicit memory phenomena and the resulting theories are limited to or primarily involve perceptual, data-driven priming, their importance may be limited. Such a restricted category of memory phenomena will probably not be very instrumental for an understanding of most forms of cognitively complex learning and problem solving. However, if conceptual priming in semantically complex processing 
tasks is shown to have the same defining characteristics of implicit memory as perceptual priming has (i.e., longlasting performance facilitation that does not depend on event recall), theories of implicit memory that incorporate these phenomena should have a much greater impact. This possibility argues for more research on conceptual priming within more complex tasks.

\section{REFERENCES}

BogartZ, R. S. (1990). Evaluating forgetting curves psychologically Journal of Experimental Psychology: Learning, Memory, \& Cognition, 16, 138-148.

BLAXTON, T. A. (1989). Investigating dissociations among memory measures: Support for a transfer appropriate processing framework Journal of Experimental Psychology: Learning, Memory, \& Cognition, 15, 657-668.

Brown, H. L., Sharma, N. K., \& Kirsner, K. (1984). The role of script and phonology in lexical definition. Quarterly Journal of Experimental Psychology, 36A, 491-506.

Carr, T. H., Brown, J. S., \& Charalambous, A. (1989). Repetition and reading: Perceptual encoding mechanisms are very abstract but not very interactive. Journal of Experimental Psychology: Learning, Memory, \& Cognition, 15, 763-778.

Clarke, R., \& Morton, J. (1983). Cross-modality facilitation in tachistoscopic word recognition. Quarterly Journal of Experimental Psychology, 35A, 79-96.

GARDINER, J. (1988). Generation and priming effects in word-fragment completion. Journal of Experimental Psychology: Learning, Memory, \& Cognition, 14, 495-501.

Hamann, S. P. (1990). Level-of-processing effects in conceptually driven implicit tasks. Journal of Experimental Psychology: Learning, Memory, \& Cognition, 16, 970-977.

JaCkson, M. D., \& MCCleliand, J. L. (1979). Processing determinants of reading speed. Journal of Experimental Psychology: General, 108, 151-181.

JACOBY, L. L. (1983). Perceptual enhancement: Persistent effects of an experience. Journal of Experimental Psychology: Learning, Memory, \& Cognition, 9, 21-38.

JACOBY, L. L., \& DALLAS, M. (1981). On the relationship between autobiographical memory and perceptual learning. Journal of Experimental Psychology: General, 110, 306-340.

JaCOBY, L. L., LeVy, B. A., \& SteinbaCh, K. (1992). Episodic transfer and automaticity: Integration of data-driven and conceptually driven processing in rereading. Journal of Experimental Psychology. Learning, Memory, \& Cognition, 18, 15-24.

Kirsner, K., Dunn, J. C., \& Standen, P. (1989). Domain-specific resources in word recognition. In S. Lewandowsky, J. C. Dunn, \& K. Kirsner (Eds.), Implicit memory: Theoretical issues (pp. 99-122). Hillsdale, NJ: Erlbaum.

Kirsner, K., Milech, D., \& Standen, P. (1983). Common and modality-specific processes in the mental lexicon. Memory \& Cognition, 11, 621-630.

KirSNER, K., \& SMITH, M. C. (1974). Modality effects in word identification. Memory \& Cognition, 2, 637-640.

KolERS, P. A. (1975). Specificity of operations in sentence recognition. Cognitive Psychology, 1, 289-306.

KOLERS, P. A. (1976). Reading a year later. Journal of Experimental Psychology: Human Learning \& Memory, 2, 554-565.

Kyllonen, P. K., Tirre, W. C., \& Christal, R. E. (1991). Knowledge and processing speed as determinants of associative learning. Journal of Experimental Psychology: General, 120, 57-79.

Levy, B. A., Barnes, L., \& Martin, L. (1993). Transfer of fluency across repetitions and across texts. Canadian Journal of Experimental Psychology, 47, 401-427.

LEvY, B. A., \& BURNS, K. I. (1990). Reprocessing text: Contributions from conceptually driven processes. Canadian Journal of Psychology, 44, 465-482.

LEVY, B. A., \& KirSNER, K. (1989). Reprocessing text: Indirect mea- sures of word and message level processes. Journal of Experimental Psychology: Learning, Memory, \& Cognition, 15, 407-417.

Lofius, G. R. (1985). Evaluating forgetting curves. Journal of Experimental Psychology: Learning, Memory, \& Cognition, 11, $397-$ 406.

Masson, M. E. J. (1986). Identification of typographically transformed words: Instance-based skill acquisition. Journal of Experimental Psychology: Learning, Memory, \& Cognition, 12, 479-488.

Masson, M. E. J. (1993). Episodically enhanced comprehension fluency. Canadian Journal of Experimental Psychology, 47, 428-465.

Masson, M. E. J., \& Freedman, L. (1990). Fluent identification of repeated words. Journal of Experimental Psychology: Learning, Memory, \& Cognition, 16, 355-373.

MORTON, J. (1979). Facilitation in word recognition: Experiments causing change in the logogen model. In P. A. Kolers, M. E. Wrolstad, \& H. Bouma (Eds.), Processing of visible language (pp. 259268). New York: Plenum.

O'Brien, R. G., \& KaISER, M. K. (1985). MANOVA method for analyzing repeated measures designs: An extensive primer. Psychological Bulletin, 97, 316-333.

RAPPOLD, V. A., \& HASHTROUDI, S. (1991). Does organization improve priming? Journal of Experimental Psychology: Learning, Memory, \& Cognition, 17, 103-114.

ROEDIGER, H. L., III (1990). Implicit memory: Retention without remembering. American Psychologist, 45, 1043-1056.

ROEDIGER, H. L., III, \& BLAXTON, T. A. (1987). Effects of varying modality, surface features, and retention interval on priming in word-fragment completion. Memory \& Cognition, 15, 379-388.

Roediger, H. L., III, Weldon, M. S., \& Challis, B. H. (1989). Explaining dissociations between implicit and explicit measures of retention: A processing account. In H. L. Roediger III \& F. I. M. Craik (Eds.), Varieties of memory and consciousness: Essays in honor of Endel Tulving (pp. 3-41). Hillsdale, NJ: Erlbaum.

Scarborough, D. L., Cortese, C., \& Scarborough, H. S. (1977). Frequency and repetition effects in lexical memory. Journal of Experimental Psychology: Human Perception \& Performance, 3, $1-17$.

SCHACTER, D. L. (1992). Understanding implicit memory: A cognitive neuroscience approach. American Psychologist, 47, 559-569.

Shimamura, A. P., \& SQuire, L. R. (1984). Paired-associate learning and priming effects in amnesia: A neuropsychological study. Journal of Experimental Psychology: General, 113, 556-570.

Shulman, A. I. (1974). Memory for words recently classified. Memory \& Cognition, 2, 47-52.

SlameCKa, N. J. (1985). On comparing forgetting rates. Journal of $E x$ perimental Psychology: Learning, Memory, \& Cognition, 11, 812816.

Sloman, S. A., Hayman, C. A. G., Ohta, N., Law, J., \& Tulving, E. (1988). Forgetting in primed fragment completion. Journal of Experimental Psychology: Learning, Memory, \& Cognition, 14, 223239.

Smith, E. R., \& Branscombe, N. R. (1988). Category accessibility as implicit memory. Journal of Experimental Social Psychology, 24, 490-504.

SRINIVAS, K., \& ROEDIGER, H. L., III (1990). Classifying implicit memory tests: Category association and anagram solution. Journal of Memory \& Language, 29, 389-412.

Tulving, E., \& SCHacter, D. L. (1990). Priming and human memory systems. Science, 247, 301-306.

WALKER, R. (1985). PLATS: Software for cognitive tasks. Unpublished computer program.

WELDON, M. S. (1991). Mechanisms underlying priming on perceptual tests. Journal of Experimental Psychology: Learning, Memory, \& Cognition, 17, 526-541.

WELDON, M. S. (1993). The time course of perceptual and conceptual contributions to word fragment completion priming. Journal of Experimental Psychology: Learning, Memory, \& Cognition, 19, 10101023

WeLdon, M. S., \& RoEDiger, H. L., III (1987). Altering retrieval demands reverses the picture superiority effect. Memory \& Cognition, $15,269-280$

Whittlesea, B. W. A. (1990). Perceptual encoding mechanisms are 
tricky but may be very interactive: Comment on Carr, Brown, and Charalambous (1989). Journal of Experimental Psychology: Learning, Memory, \& Cognition, 16, 727-730.

Wixted, J. T. (1990). Analyzing the empirical course of forgetting. Journal of Experimental Psychology: Learning, Memory, \& Cognition, 16, 927-935.

WiXTED, J. T., \& EBBESEN, E. B. (1991). On the form of forgetting. Psychological Science, 2, 409-415.

Woltz, D. J. (1988). An investigation of the role of working memory in procedural skill acquisition. Journal of Experimental Psychology: General, 117, 319-331.

WolTZ, D. J. (1990a). Decay of repetition priming effects and its relation to retention from text: A study of forgetting. Learning \& Individual Differences, 2, 241-261.

WoLTZ, D. J. (1990b). Repetition of semantic comparisons: Temporary and persistent priming effects. Journal of Experimental Psychology: Learning, Memory, \& Cognition, 16, 392-403.

WolTZ, D. J. (1995). Direct priming of conceptually driven mental operations. Manuscript submitted for publication.

Woltz, D. J., \& MADSEN, J. G. (1995). Independence in implicit and explicit memory measures for semantic processing events. Manuscript submitted for publication.

Woltz, D. J., \& ShUTE, V. J. (1993). Individual difference in repetition priming and its relationship to declarative knowledge acquisition. Intelligence, 17, 333-359.

Woltz, D. J., \& SHUTE, V. J. (1995). Time course of forgetting exhibited in repetition priming of semantic comparisons. American Journal of Psychology, 108, 499-525.

\section{NOTES}

1. The SOA of $750 \mathrm{msec}$ was set on the basis of pilot testing a range of SOA values. The pilot data indicated that overall trial performance latency on first-occurrence trials decreased as a function of increasing SOAs up to about $700 \mathrm{msec}$, after which there was no change. On the basis of these data, I assumed that $750 \mathrm{msec}$ was sufficient time for subjects to encode the first stimulus word, fully activate its meaning, and prepare for the second stimulus word.

2. This distinction between the priming of lexical and general semantic memory representations and the priming of trial-specific semantic processes corresponds roughly to the distinction between semantic and production explanations of priming made by Kirsner, Dunn, and Standen (1989).

3. The error and latency data for repeated trials in Part B are reported for all trials, regardless of the response accuracy for the corresponding trial in Part A.

4. Another difference between these two lexically primed negative trial conditions was that of response consistency between prime and target trials. However, this variable was investigated and shown to have a very minor effect on priming magnitude (Woltz, 1995).

5 . In a sample of 74 college students, there was evidence that subjects used response fluency information to make subsequent judgments about whether they had seen items before. That is, subjects were more likely to call an item old if they had just solved it with a quick and accurate response, regardless of whether it was new or old. There was no evidence that recognition performance was linked to performance facilitation beyond this effect.

(Manuscript received August 20, 1993; revision accepted for publication July 5, 1995.) 